\title{
Development of Vehicle Management Platform Based on ArcGIS
}

\author{
Mei Jing ${ }^{1,2}$, Song Hong ${ }^{1}$, Shang Wen-li ${ }^{1}$ and Zhou Xiao-feng ${ }^{1}$ \\ ${ }^{1}$ Shenyang Institute of Automation, Chinese Academy of Sciences, Shenyang, China, 110016 \\ ${ }^{2}$ University of Chinese Academy of Sciences, Beijing, China, 100049 \\ meijing@sia.cn
}

\begin{abstract}
Based on ArcGIS software, a multi-function vehicle management platform was developed, which realized functions of Vehicle electronic barrier setting, map labeling, vehicle orientation, vehicle status display, history track replay, real-time monitoring and early alarming, fault diagnosis and displaying different vehicles on multi-Screen at the same time. During the development of vehicle fleet management system ,the system architecture was built, and the system involved a number of key technology: service of data transparent transmission, query and replay history track of vehicle, and Multi -Screen display. The result suggested that the scheduling platform could display locations and state information of vehicles in a dynamic way, and have friendly interface and convenient operation.

Index Terms - Vehicle management platform; ArcGIS; real-time monitoring; Data transparent transmission; History track replay
\end{abstract}

\section{Introduction}

Fleet management platform is a vehicle-based back-office applications, it integrated vehicle data logging, satellite positioning and data communication functions for operators to provide a simple, effective method of vehicle management. ${ }^{[1]}$

Nowadays some things happened frequently, for example, vehicle are used illegally and privately, encountered traffic jams, or dispatched untimely when emergency happened. The majority of enterprises manage the fleet mainly by artificial management, lead to ill management, and the statistics is not complete. So we need to introduce a modern high-tech solution to achieve the tracking, monitoring and scheduling of vehicles ${ }^{[2-3]}$.

At present, the study of the fleet management platform and vehicle monitoring system always focus on GPS, GPRS, GIS or combines them ${ }^{[4-7]}$, the basic principle is to get the vehicle location and status information firstly by vehicle terminal which is installed in the vehicle, and then data transmit from a moving vehicle to the monitoring center by wireless network communication technology, finally Vehicle Monitoring GIS systems display location and status information of vehicles in a visual and dynamic way ${ }^{[3]}$. The relatively mainstream development way of vehicle information management platform is to choose ArcGIS Engine as the tools to develop the platform in the Visual Basic development environment ${ }^{[3,8]}$.

\section{Overall Structure}

The feature of vehicle management platform described in this article is that it can display dynamic information of different vehicles at the same time in a split-screen. We use ArcGIS as the tool to develop the platform which has B / S architecture in VS2010 development environment, as compared with the C / S mode, B / S mode is easier to centralized management and maintenance, also B / S mode have better function on ease-of-use and cross- -platform ${ }^{[9]}$. Then We call ArcGIS ArcSde, ArcgisServer, ADF, and Siverlight API to develop the vehicle management dispatch platform in MVC design model.

The architecture of vehicle management platform is shown in Figure 1. The whole platform is developed in VS2010 development environment. From Figure 1, the whole system is divided into five layers. It contains the presentation layer, service layer, business logic, data access layer and the gateway. The left side of the picture is the manifestations of every layers, which contains the browser, IIS host, WCF, read and write data service, Oracle, data receiving. Specifically, the presentation layer refers to the UI interface which is presented by the client browser, system management interface using WPF (Windows presentation foundation, which Microsoft unified for Windows subsystem) technology for development, remote user interface use Microsoft silverlight technology development. The host of service layer is IIS, and service layer enroll and schedule services through the platform news service bus. The business logic layer is to realize the WCF (Windows communication foundation, Windows Communication Interface) service. The data access layer is for data read and write through Oracle. The gateway is responsible for transferring the GPS data and CAN bus data which were received before to the database through data transparent transmission service.

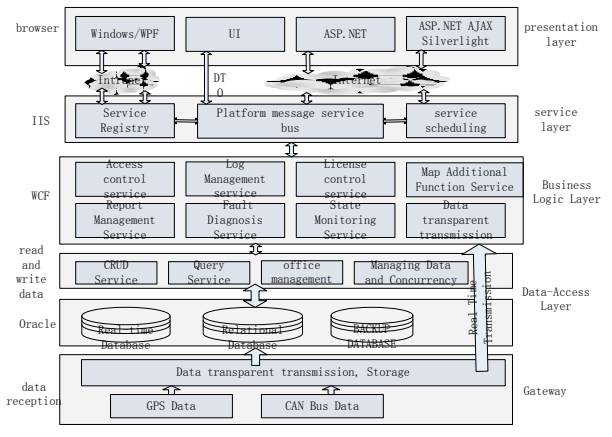

Fig. 1 Architecture of Vehicle management platform

In the whole platform, the system design by the Web Service technology and the idea of SOA (service oriented architecture), all applications access database indirectly by 
accessing WCF server, so this way enhance the integrity and security of internal data. The platform includes vehicle electronic barrier setting, vehicle orientation, status monitoring, routine maintenance, fault diagnosis, vehicle information management, report management and other services.

In the concrete implementation, we generate powerful Web applications by ASP.NET programming framework, at the same time we use a new way of ADO.NET of data accessing and Browser / Server mode. In the area of data management, GPS database using Oracle $10 \mathrm{~g}$ database to manage system, and map information database of ArcGIS is stored into Oracle through ArcSDE.

\section{Platform functions and system implementation}

\section{A. Platform Function}

The vehicle management platform has 6 function modules, including Vehicle electronic barrier setting and map labeling, vehicle status display, history track replay, real-time monitoring and early alarming, fault diagnosis and report management.

\section{1) Vehicle electronic barrier setting and map labeling}

In vehicle management platform, we not only develop a basic map display function, including zoom, zoom out, fullscreen, map hawkeye, and hierarchical display, but also set the electronic barrier, user can customize an area, and then if the vehicle traveled over the fence, the system will alarm and prompt vehicle specific information. Also we developed map labeling function, user can label information to any location on the map, and the label can add, modify, or delete, and can count the distance from one point to the other.

\section{2 ) vehicle status display}

Put the mouse on the selected vehicles, the platform will be able to display the basic information automatically ,such as the vehicle's speed, direction of travel, the latitude and longitude of the vehicle, mileage, current time.

\section{3 ) History track query and replay}

User can Type in their query to generate the trajectory that satisfy the query criteria through the history track page ; when the history track successfully generated, it can also be operated, such as pause, play, stop, fast-forward and so on. The track can be queried within one month.

\section{4) Real-time monitoring and early alarming}

The system firstly read the latest GPS data, and then display the operational status of the vehicle dynamically, at the same time vehicle symbol changes dynamically with the vehicle running direction. During the same time the alarm system will be working when the speed is too high or overrange the barrier. The real-time vehicle monitoring and early warning have 4 functions, which contains remote instrumentation, dynamic curves, dangerous driving alarming and parameter abnormality alarming.

\section{5) Fault diagnosis}

At present, a lot of literature has been studied only for single failure, the separation and estimate of multiple faults is an important problem to be solved ${ }^{[10]}$. The vehicle remote fault diagnosis module is divided into four module, which contains data acquisition module, signal processing module, fault classification module, report generation module. It not only can diagnose hardware directly related to a single physical failure, but can also analyze comprehensive faults. Diagnostic module analysis fault information by detecting data changes and read fault codes, enabling remote read fault codes, clear fault codes, read the basic functions of the data stream, and also can resolve the fault code, the meaning of the fault code and reason. In addition, diagnostic module can collect all the fault information and statistical them.

\section{6) Report management}

User can input their query conditions and then statistic data they need through by report management, the date in the report management can be vehicle classification, nature, mileage, working amount, and so on. User can also definite the report styles flexibly, report statistics items or output print.

\section{B. System implementation}

The entire system of the platform uses Web-based B / S structure design, so operation interface of the management system are browser application interface. System Application Server is Windows XP / 7 system, IIS6.0 server, framework3.5, using Oracle $10 \mathrm{~g}$ as database. Development language is $\mathrm{C}$ \#, electronic map display function is realized through by ArcGIS.

\section{1) Vehicle Location}

The definition of vehicle location is that system display position with marked picture according to the data from database, which is read by timer. Specifically We choose a map provided by ARCGIS as a base map firstly, and then add a layer on top; Next, creating point feature, which including the location and attribute information. Put these elements of these points to the corresponding layers; finally put these layers covering the base map and then vehicle positioning function is realized. As for the search of the vehicle we directly call the corresponding API provided by ArcGIS.

\section{2 ) Real-time monitoring and early alarming}

Put the mouse on the selected vehicles, the platform will be able to display speed, location, mileage, time and other information of the vehicle automatically. Meanwhile, according to the selected the number of vehicle , user can find the path from the database ,futher we will get a polygonous buffer, and then we can contrast every coordinate point we get with the polygon, If the point is in the polygonous, suggesting that it is in bounds; if it is not, suggesting that it is crossborder, system will alarm save the information in the database.

\section{3 ) Historical track query and replay}

Firstly we use the WebService items we added in the system before, connecting to the database and getting the functional code we required. Secondly we add Service reference, call function from WebService in MainPage.xaml.cs. Thirdly we set the DispatcherTimer adding 
a point in GraphicsLayer every $2 \mathrm{~s}$, and then system will generate the historical track.

\section{Key Technologies}

\section{A. Data transparent transmission service}

Firstly system transfer GPS data and CAN bus data to data transparent transmission service module which is obtained from the terminal equipment, after the data is processed, part of the data is sent to the database directly, and the other part of the data is transferred to data transparent transmission service module to be encapsulation, passing to the upper service module as the way of object flows, and then the data is realtime displayed visualizations . Architecture of data transparent transmission is shown in Figure 2. Through the way of data transparent transmission, we guarantee the timeliness, reliability and accuracy of real-time vehicle status information maximum.

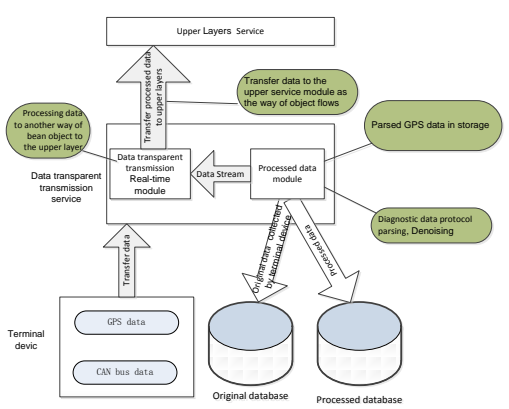

Fig. 2 Diagram of data transparent transmission

\section{B. Historical track query and replay}

Specific implementation methods of the historical track query and historical track playback function, respectively to be called in The class VehicleTrack.aspx , ForwardTrack , StopTrack, PlayTrack and PauseTrack. We use reasonable and effective algorithm in generating vehicle history track, which allowing users to query the vehicle within $24 \mathrm{~h}$, its response time is less than guarantee or even lower than $1 \mathrm{~s}$. We use reasonable and effective algorithm in generating vehicle history track, which allowing users to query the vehicle within $24 \mathrm{~h}$, its response time is less than guarantee or even lower than $1 \mathrm{~s}$. In the algorithm we choose the feature data of generated historical trajectory data from a large number of vehicle history data, to reduce the space of generate trajectory calculation. The algorithm described below.

1) Putting the moment to as the starting position adding to the track, updating the latest traveling direction $\mathrm{d}$ (saved) of the track as the vehicle traveling direction of the moment t0.

2) Reading a GPS data in chronological order, if the GPS position is the endpoint, perform step 5), generating end track algorithm, otherwise step 3 ).

3 ) If the point is the corner of the road junction or polyline road point, step 5); otherwise step 4).

4 ) Comparativing GPS data recorded in the vehicle traveling direction $\mathrm{d}$ (current) and $\mathrm{d}$ (saved) the difference, if $\mathrm{d}$ (current)-d (saved) $\mid>\delta$, where $\delta$ is the threshold value, then perform step 5); otherwise, perform step 1).
5) Adding the position information from the GPS data to the track, updating the latest traveling direction d (saved) as the traveling direction d (current) which is recorded in GPS data.

\section{Split screen monitor}

While Vehicle monitoring, user can use the right-click menu list to select full-screen exit, split-screen mode selection and the vehicle which is monitored by current screen in the window of each screen, also user can monitor vehicles by query conditions at the top of each screen. During Split-screen monitoring, platform will display multiple screens selected by user, each screen is a separate event handler. Vehicles on each of the screen can be selected to be queried simultaneously. We use CustomPanel class programmed by Silverlight to realize Split-screen monitor. when the user is operating, they only need to enter the number of split-screen, then the multiple screens can be displayed in accordance with the user input.

\section{Conclusion}

In this thesis, we research the vehicle management platform based on ArcGIS, using C \# language and ArcGIS controls to develop the vehicle management application platform. The function of platform is to achieve real-time dynamic positioning of the vehicle, monitoring, scheduling, enabling the visualization of vehicle monitoring, dispatching and control; Furthermore it has the characteristics of map labeling, split-screen display and so on. This research change the past vehicle command and dispatch mode, which is easier interfered by human, providing a new vehicle management tools for people, which improve the vehicle monitoring and dispatching management capabilities of transport sector and enterprises.

\section{References}

[1] Johan Fagerberg, Tobias Ryberg. Fleet management and wireless M2M[M]. Berg Insight AB, March 2010.

[2] Lei Yu-hai, Wang Yu. Research and development of vehicle fleet management information system software[J]. Traffic Science Technology and Economy, 2004(3):53-54.

[3] Wu Jian-hua. Development of Vehicle monitor GIS system based on ArcGIS Engine[J]. Geo-Information Science, 2011(1):88-94.

[4] Chen Yi-pei, Design and development of vehicle monitor management system based on GPS/GIS/GPRS[D]. Xi'an: Xi'an University of Technology, 2009.

[5] He Li-hong. Design and realization of vehicle monitoring subsystem based on GIS / GPS[D]. Beijing: Beijing Jiaotong University, 2009.

[6] Wang Jun, Hu Ping, Shi Tao. Implementation of vehicle monitoring system based on GIS / GPS / GPRS[J]. Microcomputer Information, 2006 (9): 290-292.

[7] Zhang Shu-bi, Liu Zuo-cai. as before. Design and realization of GPS vehicle monitoring system based on GIS [J]. Bulletin of Surveying and Mapping, 2002 (6):31-33.

[8] Li Chun-li, Zeng Zhi-yuan, Xu Xue-jun. Vehicle monitoring system based on ArcGIS Engine[J]. Computer Engineering, 2006, 32 (24): 257259.

[9] Luo Ling. Vehicle scheduling system based on .NET component[J]. Computer Engineering, 2008, 34 (11): 275-277.

[10] Jia Qing-xian, Zhang Ying-chun, Guan Yu. Fault diagnosis of nonlinear systems based on analytical models : A Survey[J]. Information and Control, 2012, 41(3): 356-364. 